\title{
FARM OUT THE RESPONSIBILITY
}

by Bernard Dixon

$\mathbf{T}$ he most astutely sensitive means of monitoring rare side-effects of newly introduced medicines is by asking patients themselves to report any odd quirks, rashes, or rumbles they may experience after starting a novel therapy. Rather than relying on busy physicians to spot such perturbations during routine consultations, or trusting in the (mythical) Olympian awareness of regulatory bodies, we do well to harness the senses and full-time assistance of those who know their own bodies most intimately of all. Someone with a lifetime's experience of the vagaries of his or her own skin tone, digestion, and bowel habits is far more likely than any third party to pick up significant changes if and when they occur.

A similar approach could well be carried over into a very different domain, to ensure close surveillance over the performance of genetically modified organisms (GMOs) in the environment. Clearly, no committee on Earth, however sage-like in intellect or catholic in composition, could hope to anticipate every conceivable consequence of a deliberate release. Equally unrealistic is the prospect of establishing dedicated national or indeed international bodies to monitor exhaustively in space and time the behavior of each and every GMO that is disseminated in the field. Why, then, should we not place the responsibility for noting and reporting unexpected repercussions of such releases on those most directly involved-on the farmers who plant out new varieties and use novel microbial inocula on their land? Surely their day-by-day, year-by-year practical experience fits them better than anyone for such a scrutinizing role?

This, essentially, was the idea floated by John Beringer, chairman of the U.K.'s new Advisory Committee on Releases to the Environment, while addressing a symposium at the Royal Society of Edinburgh, held during the recent Edinburgh International Science Festival. Beringer was speaking about one specific problem-that of weediness in plants. He was also discussing what form of surveillance machinery should be established in future, when assessment committees have dispensed with their current requirements for detailed monitoring. Nevertheless, this is an approach that could have wider utility in bringing to light other unwelcome consequences of deliberate release.

One particular adverse consequence, which Beringer identified as "potentially the greatest risk," would be the acquisition of herbicide resistance genes by modified plants, making them less easy to control in subsequent crops. "The potential for such problems already exists with traditional breeding and the introduction of new crops into a region," he said. "Perhaps therefore the most valuable monitoring would be by farmers themselves, who might be expected to report such problems as a condition of using new varieties, whether they are manipulated or not."

Longstanding contact with the land, combined with considerable quantitative and qualitative knowledge of vari- ations in harvest from season to season and the responses of particular crops to various agrochemicals, surely makes farmers the ideal observers to monitor the performance of at least some GMOs. Given that much of their craft is dedicated to the obliteration or management of weeds, spotting the emergence of weediness in modified plants or the transfer of weedy traits to non-modified varieties would seem to be a task for which they are uniquely well qualified.

Equally important is the need for vigilance in detecting unforeseen migrations of introduced plants into terrain where they could prove a nuisance. Several recent reports have indicated that oilseed rape, cultivation of which has increased considerably over the past decade in Britain, may be migrating into and colonising disturbed habitats. Although the varieties used have not been genetically altered, and although the evidence so far is minimal, these claims highlight a potentially serious difficulty in the case of manipulated plants. Who better than a farmer to record and report movements of this sort?

A second conjectural hazard of novel plants came to light as a result of the deliberations of a GENHAZ working party associated with the U.K. Royal Commission on Environmental Pollution, which two years ago issued a report on deliberate release. Adopting an approach close to the HAZOP (Hazard and Operability) methodology used to assess the safety of particular operations in the chemical industry, the GENHAZ group set out to catalogue all conceivable consequences of the production and release of GMOs. One possibility, not previously addressed, was that an introduced gene, as well as fulfilling its intended purpose, might be expressed in the pollen grains of a manipulated plant, making them more likely to provoke hay fever in susceptible individuals. Cloned gene products in pollen might also pollute honey and cause harm to bee larvae.

Again, there has been some anecdotal evidence in the U.K. that could support fears of this sort, with reports of heightened symptoms in hay fever sufferers in areas where oilseed rape has been cultivated widely for the first time. It remains to be seen whether this confirms that the pollen is unusually allergenic, whether the problem is widespread or serious, and indeed whether the claims are actually true at all. But again, this is precisely the type of effect on which farmers might be expected to be able to provide early warnings.

As Beringer pointed out in Edinburgh, some aspects of possible gene transfer from manipulated organisms cause quite disproportionate anxiety-especially when placed alongside natural transfers of genes for such toxic compounds as ricin and alkaloids, which attract no public interest whatever. At the same time, there is still insufficient research upon which to assess selective advantages conferred on plant populations through pollen carrying novel genes. We shall hear a good deal more about this, and about means of tackling the problem. 